\title{
A Novel Calibration Method for Signal Leakage between Multiple Channels in Array Imaging
}

\author{
Zhenmao Cui ${ }^{1,2} \mathbb{D}$, Binbin Cheng ${ }^{1,2, *}$, Xianjin Deng ${ }^{1,2}$, Qiang Wu ${ }^{1,2}$, Jianfei An ${ }^{1,2}$ and \\ Yang Yu ${ }^{1,2}$ \\ 1 Institute of Electronics Engineering, China Academy of Engineering Physics, Mianyang 621900, China; \\ cuizhenmao@mtrc.ac.cn (Z.C.); Dengxianjin@mtrc.ac.cn (X.D.); wuqiang@mtrc.ac.cn (Q.W.); \\ anjianfei@mtrc.ac.cn (J.A.); yuyang@mtrc.ac.cn (Y.Y.) \\ 2 Microsystem and Terahertz Research Center, China Academy of Engineering Physics, \\ Chengdu 610200, China \\ * Correspondence: Chengbinbin@mtrc.ac.cn
}

Received: 14 August 2020; Accepted: 27 September 2020; Published: 2 October 2020

\begin{abstract}
In this paper, the array imaging process with the presence of signal leakage between channels is derived and analyzed in short-range imaging applications. The echo wave of the system can be divided into two parts: the echo signals we expected, and the echo signal that resulted from the leaked signals. Signals leaked to the adjacent channels can lead to ghosts which can deeply deteriorate the system cross resolution. To solve this problem, a concise calibration method is proposed to remove the impacts of the signal leakages on the imaging quality. Furthermore, the results based on the simulation and the real-world data validate the effectiveness of the proposed method, which indicates that this calibration method can mostly cancel the influence of the signal leakages.
\end{abstract}

Keywords: array imaging; signal leakages; multichannel systems; calibration method

\section{Introduction}

Nowadays, near-field millimeter-wave imaging has been widely applied to various fields such as human body security [1-7], 3-D body size measurement [8-10], and skin cancer detection [11-13]. However, there are always some hardware defects in the system which can deeply deteriorate the system's performance, especially in array imaging. Therefore, to get a high-quality image of these radar systems, some calibration processes must be done before imaging. A variety of calibration methods for different system problems have been proposed in the past, such as computational correction algorithm for nonlinearities [14-16], phase error correction [17-19], and channel imbalance correction [20,21].

Signal leakage is an important problem which needs to be processed in a radar system. Signal leakage that occurs between transmitter and receiver can cause a strong degradation of the detection sensitivity as a result of the phase noise of the transmit oscillator. Its calibration method has been widely discussed. There are several ways to deal with this leakage. One way is to increase the distance between the Tx and the Rx to reduce the leakage [22]. The second way is to add a closed loop leakage canceller which can generate an error vector including leakage amplitude and phase information, and this information can be used to carry out the calibration [23-25].

Signal leakage between different channels in multichannel systems is a unique problem, especially in imaging systems with requirements of high resolution and sensitivity. The leakage that occurs between the transmitter and the receiver has been widely studied. There are a lot of discussions about its signal model and processing method [26-28]. However the impact of the leakage that occurs between transmitters or receivers has rarely been discussed. In this paper, the array imaging process with the presence of signal leakage caused by the lower isolation between transmitters or 
receivers is researched. First, a common array structure and its signal leakage model between different channels are introduced. Second, the imaging process with the presence of signal leakage is derived based on the model and the influence of the signal leakage is discussed. Signals leaked to adjacent channels can lead to ghosts which can deeply deteriorate the system cross resolution. To eliminate the bad influence of the signal leakages, a calibration method operating in the beam domain is proposed next. Finally, to validate the effectiveness of the proposed method, a simulation and some experiments are carried out. All the derivation and analysis are based on a common array structure applied in short range imaging.

The paper is structured as follows. Section 2 introduces the used array structure and signal leakage model between different channels. The impacts of the channel signal leakages are analyzed in Section 3. Based on the analysis, a calibration method is prosed in Section 4. Finally, in Section 5, we verify the calibration method with a simulation and some experiments.

\section{Array Structure and Signal Leakage Model}

Figure 1 is a common antenna array structure of the millimeter wave in short range imaging [29]. The architecture of the array consists of two linear arrays. Suppose that the transmitting array is on the above and the receiving array is on the below. The interval between the transmitting or receiving elements is $\lambda$. In order to get equivalent phase centers with an interval of $\lambda / 2$, the receiving array is shifted $\lambda / 2$ along the $x$ direction with respect to the transmitting array. When one transmitting element is working, its two adjacent receiving elements carry out the signal reception in sequence. The signal is usually regarded as though it is transmitted and received both at the midpoint of the line connecting the corresponding working antennas. Thus, a set of equivalent phase centers spaced $\lambda / 2$ can be obtained.

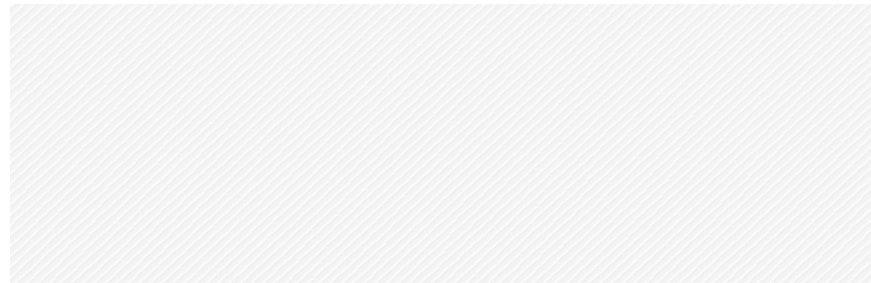

Figure 1. Array configuration of the transmitting and receiving elements.

In order to implement the signal's independent emission and reception of the array units described in Figure 1, the imaging system usually adopts a multistage switch matrix. Figure 2 is a common switch network structure. For the convenience of description, a three-stage switch matrix is used in Figure 2. The first, second, and third stage of the transmitting network adopt SPTK, SPTM, and SPTN switch chips, respectively, as shown in Figure 2. The structure of the receiving switch network is the same as the transmitting. Therefore, this architecture can implement a $1 \times \mathrm{K} \times \mathrm{M} \times \mathrm{N}$ switch network.

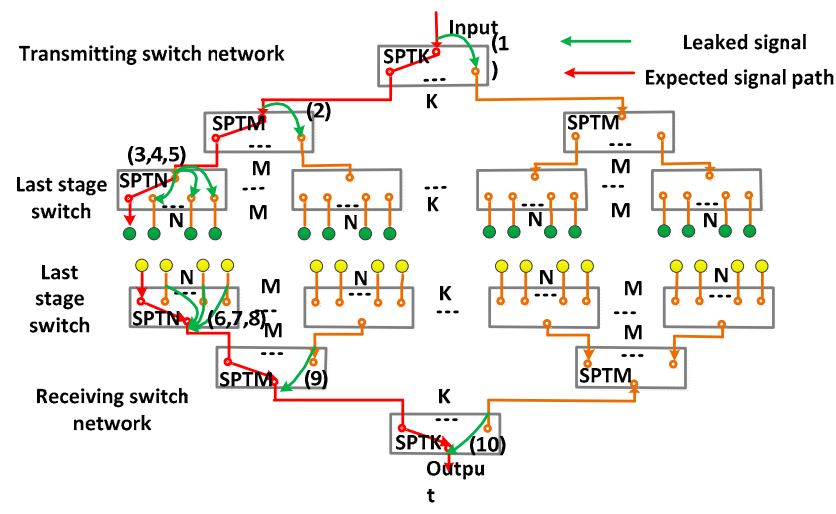

Figure 2. RF switch network. 
Based on the structure, we can make a simple analysis about the signal leakages occurred in the switch network due to the lower isolation of the switch chips when the signal is transmitted and received. Suppose that the red path in Figure 2 is the signal path we expected; therefore, the corresponding switch channels are opening while the other channels on the same chip are closed at the same time. As the isolation of the switch is limited, some signals are leaked to the closed channels, indicated by the green lines in Figure 2. The signal leakages occur in every switch stage, but those which do not occur at the last stage do not need to be considered because they have been attenuated at least twice when they arrive at the antenna and their impacts to the imaging result are not significant. Thus, only the signal leakages that occurred at the same switch chip with the opened channel at the last stage need to be considered. That means only the $N-1$ channels' signal leakages need to be considered on the transmitting switch array or the receiving array at a certain moment.

Because the signal leaked by the transmitting array and the receiving array in the received signal at a certain time is linearly superimposed, the analysis method in this paper, such as Fourier transform, has linear properties. Therefore, in order to simplify the analysis, we can independently analyze the signal leakage's influence of the transmitting array and the receiving array. Because the two analysis processes are similar, only the signal leakage of the transmitting array is considered, and an assumption is made that signal leakage does not exist between the receiving switch matrixes. Under this assumption, for the signal transmitted from the expected transmitting antenna and the signals leaked from the adjacent antenna, there are two sets of paths to the receiving antenna, as Figure 3 shows. In one set of paths (indicated by green and red lines in Figure 3), the signals illuminate the targets and then are reflected to the receiving antenna. In the other set of paths (indicated by yellow line in Figure 3), the signal is directly leaked to the receiving antenna without the reflection of the targets, which could be called the $\mathrm{Tx} / \mathrm{Rx}$ coupling interference. The Tx/Rx coupling interference has a huge impact on the imaging quality. It usually has a higher power level compared to the target echo wave such that the phase noise occurred with it sometimes may raise up the system noise floor. It will deteriorate the dynamic range of the imaging system. However, the noise could be cancelled mostly by using the empty background noise. As such, the Tx/Rx coupling interference would not be concerned in this paper. Only the signals (original signal or the leaked signals) reflected by the targets are concerned.

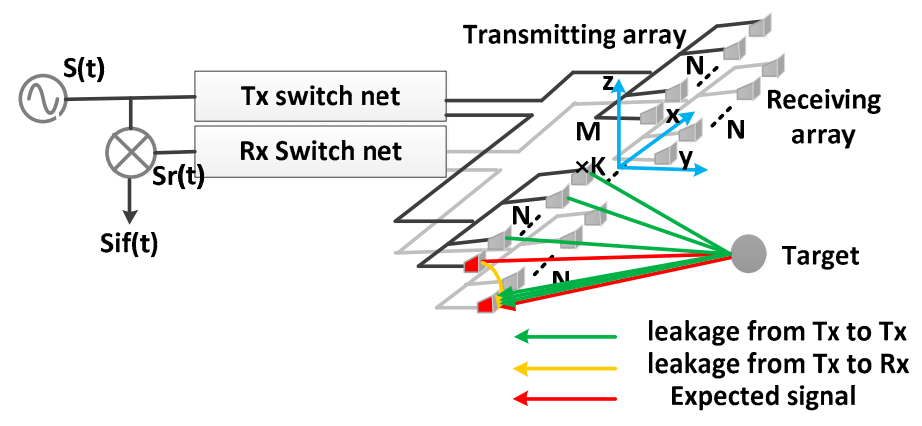

Figure 3. Signal path form Tx to Rx.

Suppose that the $n$th transmitting element's position is $\left(x_{n}{ }^{T x}, 0, z_{a}\right)$ where $\mathrm{n} \in\{1,2, \ldots, \mathrm{K} \times \mathrm{M} \times \mathrm{N}\}$. The $\mathrm{m}$ th receiving element's position is $\left(x_{m}{ }^{R x}, 0,-z_{a}\right)$ where $\operatorname{m}\{\{1,2, \ldots, \mathrm{K} \times \mathrm{M} \times \mathrm{N}\}$. The target position is $(\mathrm{x}, \mathrm{y}, 0)$. The flight trip from the $n$th transmitting element to the target is:

$$
R_{n}^{T}=\sqrt{\left(x-x_{n}^{T x}\right)^{2}+y^{2}+z_{a}^{2}}
$$

and from the target to the $m$ th, the receiving element is:

$$
R_{m}^{R}=\sqrt{\left(x_{m}^{R x}-x\right)^{2}+y^{2}+z_{a}^{2}}
$$


Then, the echo wave when the $n$th transmitting element and the $m$ th receiving antenna is working can be expressed as:

$$
s_{i f}(k, m, n)=\sigma \exp \left(j k\left(R_{n}^{T}+R^{R}{ }_{m}\right)\right)+\eta \sum_{i \in \Phi_{(n)}} \sigma \exp \left(j k\left(R_{i}^{T}+R_{m}^{R}\right)\right)
$$

where:

$$
\Phi_{(n)}=\left\{\begin{array}{l}
\left(\left[\frac{n}{N}\right]-1\right) \cdot N+1:\left[\frac{n}{N}\right] \cdot N \text { if } \operatorname{rem}(n, N)=0 \\
{\left[\frac{n}{N}\right] \cdot N+1:\left(\left[\frac{n}{N}\right]+1\right) \cdot N \text { if } \operatorname{rem}(n, N) \neq 0}
\end{array}\right.
$$

which represents the transmitting element' position set of the leaked signals. $[A]$ represents the nearest integer less than or equal to $A$. $\operatorname{Rem}(n, N)$ represents the remainder after division of $\mathrm{n}$ by $N$. $\lambda$ is the wavelength. $k$ is the wavenumber $2 \pi / \lambda$. $\eta$ is the attenuation coefficient of the leaked signal compared to the original signal (suppose that the original signal amplitude is 1 ). $\sigma$ is the radar scattering coefficient (RCS) of the target. The echo wave consists of two parts. The first term represents the echo signal we expected, and the second term represents the echo signals that result from the leaked signals.

It is difficult to make a fast imaging calculation according to Equation (1). If the distance between the transmitting element and the receiving element is very close, then some phase center approximation can be made according to the monostatic measurement method developed by Kell [30] and we can approximate that the signal is transmitted and received at the same place at the equivalent phase center located at the midpoint of the transmitting and receiving antenna connection. The echo wave can be expressed as:

$$
s_{i f}(k, m, n)=\sigma \exp \left(-j 2 k \sqrt{\left.\left(x_{t}-\left(x_{n}^{T x}+x_{m}^{R x}\right) / 2\right)^{2}+y_{t}^{2}\right)}+\eta \sum_{i \in \Phi} \sigma \exp \left(-j 2 k \sqrt{\left(x_{t}-\left(x_{i}^{T x}+x_{m}^{R x}\right) / 2\right)^{2}+y_{t}^{2}}\right)\right.
$$

According to Equation (1), the elements of the set $\Phi$ are integers in the range of $n-N+1$ to $n+N-1$ according to the configuration of the array described earlier. However, there are only $N-1$ elements in the set $\Phi$, not $2 N-1$. As such, the $\Phi$ 's elements cannot traverse all of these numbers from $n-N+1$ to $n+N-1$ at a specific transmitting and receiving position. Its elements change with the transmitting element's position. Therefore, the second term of echo Equation (2) varies with the transmitting element position. It is difficult to make a direct and clear analysis by using it. The first term can be regarded as a special case of the second term when $I=n$ and $\eta=1$, so we can merge the first and the second items. Based on the above reasons, we rewrite the echo wave Expression (2) as follows:

$$
s_{i f}(k, m, n)=\sum_{i=n-N+1}^{i=n+N-1} u_{i}(n) \cdot \eta_{i} \cdot \sigma \cdot \exp \left(-j 2 k \sqrt{\left(x_{t}-\left(x_{i}^{T x}+x_{m}^{R x}\right) / 2\right)^{2}+y_{t}^{2}}\right)
$$

where

$$
u_{i}(n)=\left\{\begin{array}{cc}
1 & \text { if } i \in \Phi \text { or } i=n \\
0 & \text { other }
\end{array} \text { and } \eta_{i}=\left\{\begin{array}{cc}
\eta & \text { if } i \in \Phi \\
1 & \text { if } i=n \\
0 & \text { other }
\end{array}\right.\right.
$$

Set that $\left(x_{n}{ }^{T x}+x_{m}{ }^{R x}\right) / 2=x_{a}$, considering that the transmitting interval is $\lambda$ according to the system model shown in Figure 1, then we can get $\left(x_{i}^{T x}+x_{m}{ }^{R x}\right) / 2=\left(x_{i}-n+n^{T x}+x_{m}{ }^{R x}\right) / 2=x_{a}+\lambda / 2(i-n)$. Using this relation, the echo Expression (3) can be rewritten to:

$$
s_{i f}\left(k, x_{a}\right)=\sum_{i=n-N+1}^{i=n+N-1} u_{i}(n) \cdot \eta_{i} \cdot \sigma \cdot \exp \left(-j 2 k \sqrt{\left(x_{t}+(i-n) \cdot \lambda / 2-x_{a}\right)^{2}+y_{t}^{2}}\right)
$$

In this section, a common array structure applied in short range imaging is introduced. Its signal model with the presence of signal leakage is constructed as Expression (4). Based on the expression, we can do further analysis about the impacts of the signal leakage on array imaging. 


\section{The Analysis of the Signal Leakage Impact on Array Imaging}

Image reconstruction can be made based on Expression (4). Firstly, one-dimensional Fourier transform is implemented along the variable $x_{a} . \eta_{i}$ and $\sigma$ would not change with $\mathrm{n}$, so we can obtain the wavenumber distribution of the echo data as follows:

$$
s_{i f}\left(k, k_{x}\right)=\sum_{i=n-N+1}^{i=n+N-1} U_{i} \cdot \underbrace{\left[\eta_{i} \cdot \sigma \exp \left(-j k_{x}\left(x_{t}+(i-n) \cdot \lambda / 2\right)-j\left(\sqrt{4 k^{2}-k_{x}^{2}}\right) y_{t}\right)\right]}_{A}
$$

$U_{i}$ is the Fourier transform of $u_{i}(n)$. When $i=n, u_{n}=1, u_{i}(n)$ is a constant, its spectrum is a $\delta$-function. When $i \neq n, u_{i}(n)$ is a periodic rectangular pulse function of receiving number $\mathrm{n}$ due to the special switch matrix configuration of the array and its value is between 0 and 1 . Its spectrum $U_{i}$ is shown in Figure 4, which can be expressed as:

$$
U_{i}=\sum_{j} S a(j \pi \tau / T) \delta\left(k_{x}-2 j \pi / T\right)
$$

where $j=0, \pm 1, \pm 2, \ldots, S a(x)=\sin (x) / x, \tau$ is the pulse width of $u_{i}(n), \mathrm{T}$ is the period of the $u_{i}(n) . U_{i}$ can be seen as some $\delta$-function combination with the envelope of $S a(j \pi \tau / T)$.
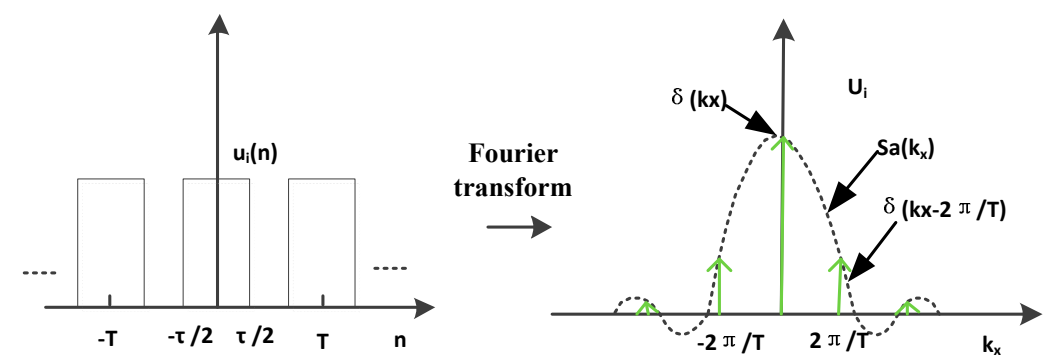

Figure 4. The Fourier transform of $u_{i}(n)$.

We can see in Figure 4 that the power of other $\delta$-function $\left(\delta\left(k_{x}-\Delta k_{j}\right), \Delta k_{j} \neq 0\right)$ is weaker compared to the $\delta\left(k_{x}\right)$ because the value of $S a\left(k_{x}\right)$ decreases sharply as $k_{x}$ increases. It would not have an important influence on the image. $\delta$-functions $\left(\delta\left(k_{x}-\Delta k_{j}\right), \Delta k_{j} \neq 0\right)$ will be ignored in the following derivation, namely $U_{i} \approx \delta\left(k_{x}\right) \cdot \gamma_{i}$ is the ratio of the amplitude of $\delta\left(k_{x}\right)$ in $U_{i}$ to the $\delta\left(k_{x}\right)$ in $U_{n}$. Obviously, $\gamma_{n}=1$. The convolution result of $\delta\left(k_{x}-\Delta k_{j}\right)$ with $\mathrm{A}\left(k_{x}\right)$ is still $\mathrm{A}\left(k_{x}-\Delta k_{j}\right)$. Then, the wavenumber distribution (5) can be simplified as follows:

$$
s_{i f}\left(k, k_{x}\right) \approx \sum_{i=n-N+1}^{i=n+N-1} \gamma_{i} \cdot \eta_{i} \cdot \sigma \exp \left(-j k_{x}\left(x_{t}+(i-n) \cdot \lambda / 2\right)-j\left(\sqrt{4 k^{2}-k_{x}^{2}}\right) y_{t}\right)
$$

Set $k_{y}=\sqrt{4 k^{2}-k_{x}^{2}}$, Expression (6) can be simplified as:

$$
\mathrm{s}_{i f}\left(k_{y}, k_{x}\right) \approx \sum_{i=\mathrm{n}-\mathrm{N}+1}^{i=n+N-1} \gamma_{i} \cdot \eta_{i} \cdot \sigma \cdot \exp \left(-j k_{x}\left(x_{t}+(i-n) \cdot \lambda / 2\right)-j k_{y} y_{t}\right)
$$

It is easy to find that the phase of $S_{i f}\left(k_{y}, k_{x}\right)$ is a linear combination of variable $k_{x}$ and $k_{y}$. Thus, applying a 2D-FFT or 2D-IFFT on it, the image result will be obtained. One thing to notice is that the map from $k$ to $k_{y}$ is not a linear process, therefore, the $k_{y}$ directly achieved form the uniformly sampled $k$ is not equally spaced. Before the 2D-IFFT, a process called Stolt interpolation [31,32] needs to be 
carried out which will not be discussed in this paper. Suppose that the band of the $k_{x}$ and $k_{y}$ is $B_{x}$ and $B_{y}$, respectively. Then, the final image result is obtained as follows after 2D-IFFT:

$$
P_{\text {original }}(x, y) \approx \sum_{i=n-N+1}^{i=n+N-1} \gamma_{i} \cdot \eta_{i} \cdot \frac{2 \sin \left(\frac{B_{x}}{2} \cdot\left(x-\left(x_{t}+(i-n) \cdot \lambda / 2\right)\right)\right)}{x-\left(x_{t}+(i-n) \cdot \lambda / 2\right)} \cdot \frac{2 \sin \left(\frac{B_{y}}{2} \cdot\left(y-y_{t}\right)\right)}{y-y_{t}}
$$

Set that

$$
P(x, y) \approx \frac{2 \sin \left(\frac{B_{x}}{2} \cdot\left(x-x_{t}\right)\right)}{x-\left(x_{t}+(i-n) \cdot \lambda / 2\right)} \cdot \frac{2 \sin \left(\frac{B_{y}}{2} \cdot\left(y-y_{t}\right)\right)}{y-y_{t}}
$$

which can be seen as the ideal point spread function of the system. The $P_{\text {original }}(x, y)$ can be rewritten as:

$$
P_{\text {original }}(x, y) \approx \sum_{i=n-N+1}^{i=n+N-1} \gamma_{i} \cdot \eta_{i} \cdot P(x-(i-n) \cdot \lambda / 2, y)
$$

It is obvious that the original point spread function $P_{\text {original }}(x, y)$ influenced by the leaked signals is a combination of the $P(x, y)$ shifted $(i-n) \lambda / 2$ in the $x$ direction. This can deteriorate the system cross resolution. It is easy to imagine that the superposition of several pulses is usually wider than a single pulse. The larger the coefficient $\gamma_{i}$, the more serious the deterioration of the cross resolution is. On the contrary, the leaked signals have little impact on the range resolution according to the $P_{\text {original }}(x, y)$ expression.

In this section, the imaging process is derived. The final imaging result is conveyed as Expression (10). The influence of the signal leakage proposed above is analyzed. It can deteriorate the system cross resolution but have little impact on the range resolution.

\section{Calibration Method of the Signal Leakage}

To eliminate the bad influences of the signal leakages, a calibration method will be proposed next. The shift in the time domain can be compensated in the frequency domain. Doing 1-D FFT along the $x$ direction on both side of the Expression (10), the following expression can be obtained:

$$
F F T_{x}\left(P_{\text {original }}(x, y)\right) \approx \sum_{i=n-N+1}^{i=n+N-1} \gamma_{i} \cdot \eta_{i} \cdot F F T_{x}(P(x, y)) \cdot e^{-j \cdot k_{x} \cdot(i-n) \cdot \lambda / 2}
$$

Then, the ideal PSF can be obtained:

$$
P(x, y) \approx \operatorname{IFFT}_{x}\left(F F T_{x}\left(P_{\text {original }}(x, y)\right) / \sum_{i=n-N+1}^{i=n+N-1} \gamma_{i} \cdot \eta_{i} \cdot e^{-j \cdot k_{x} \cdot(i-n) \cdot \lambda / 2}\right)
$$

The denominator in the IFFT $x$ parenthesis can be regarded as a calibration factor in $k_{x}$ domain. We call it array calibration factor. It makes the ghost caused by signal leakages shift to the original position. The factor can be obtained according to the configuration of the switch array. When the calibration factor is ready, the calibration can be implemented according to Expression (11) in the frequency domain. Since the calibration factor is determined by the structure of the array, this calibration method can be named array calibration factor method (ACFM).

The calibration process can be carried out according to the following steps:

Step 1: Measure the attenuation coefficient $\eta$ of the antenna array, then the coefficient $\eta_{i}$ can be obtained. Step 2: Calculate the ratio $\gamma_{i}$ according to Expression (6). It is usually difficult to obtain its analytical form; however, we can get its numerical solution through some simulation according to the application scenario of the antenna array. 
Step 3: Calculate the array calibration factor $\sum_{i=n-N+1}^{i=n+N-1} \gamma_{i} \cdot \eta_{i} \cdot e^{-j \cdot k_{x} \cdot(i-n) \cdot \lambda / 2}$ by using result of Step 1 and Step 2.

Step 4: Calculate the calibration result $P(x, y)$ by using $P_{\text {original }}(x, y)$ according to Expression (11).

Because the structure and isolation of the antenna array usually do not change, coefficient $\eta$ and ratio $\gamma_{i}$ are constants. The calibration factor in Step 3 only needs to be calculated once. The computational complexity of the calibration method proposed above is only related to Step 4. If the dimension of $P(x, y)$ is $N_{x} \times N_{y}$, the computational complexity of Step 4 is $\mathrm{O}\left(N_{x}, N_{y}\right)$. It has no significant effect on the imaging speed.

In this section, a calibration method operating in the beam domain is proposed. Its calibration steps and computational complexity are introduced. It has no significant effect on imaging speed.

\section{Simulation and Experiment}

\subsection{Simulation Results}

Suppose that the configuration of the switch array is $K=5, M=4$, and $N=4$. The operating frequency of the system is $27-33 \mathrm{GHz}$ and the isolation of the switch is $10 \mathrm{~dB}$. Then, the switch array can match with a $1 \times 80 \mathrm{Tx}$ 's antenna array and a $1 \times 80 \mathrm{Rx}$ 's antenna array. The point spread function of the array is simulated. The imaging results are shown in Figures 5 and 6 . Figure $5 a, b$ are the PSF when the signal leakages between different channels don't exist and exist, respectively. Figure 6 shows the comparison of the cross range PSF and the comparison of the range PSF in the two different cases, respectively. It is obvious that the signal leakages between different channels make the PSF broaden in cross range direction but it has little effect on the PSF in range direction. According to Expression (10), the PSF $P_{\text {original }}(x, y)$ influenced by leaked signals is a combination of the $P(x, y)$ shifted $(i-n) \lambda / 2$ in the $x$ direction but not shift in the $y$ direction. The result of the superposition of several pulses with misaligned centers will be wider than the pulse before superposition, thus, the system cross resolution along the $\mathrm{x}$ axis is deteriorated and the range resolution along $\mathrm{y}$ axis is not affected. We can see that in Figure 6, the cross resolution of signal leakage is worse, and the range resolution does not change compared to the results without signal leakage. It is consistent with the analysis above.

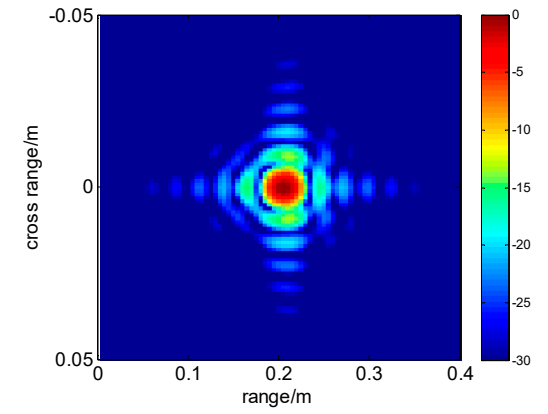

(a)

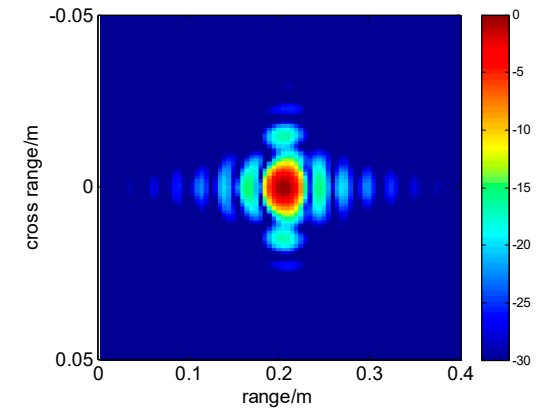

(b)

Figure 5. Simulated PSF in two different cases, (a) is the PSF when the signal leakages do not exist and (b) is the PSF when leakages exist. 


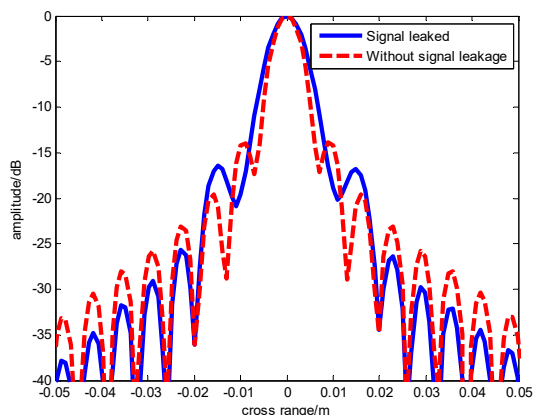

(a)

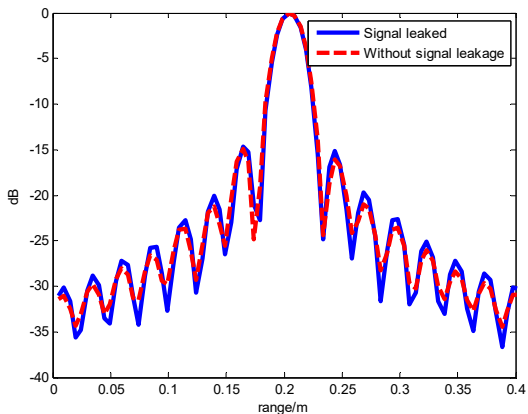

(b)

Figure 6. The comparison of PSF in two different dimensions, (a) is the cross range PSF and (b) is the range PSF.

To eliminate the influence of the signal leakage, a calibration was made according to Expression (11). The array calibration factor is shown in Figure $7 \mathrm{a}$ and the calibration results are shown in Figures $7 \mathrm{~b}$ and 8 . The imaging result after calibration is basically the same as the result without the signal leakages. It indicates that the calibration method can eliminate the effects of leakage signals.

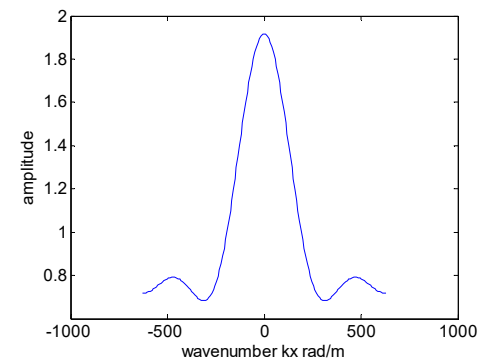

(a)

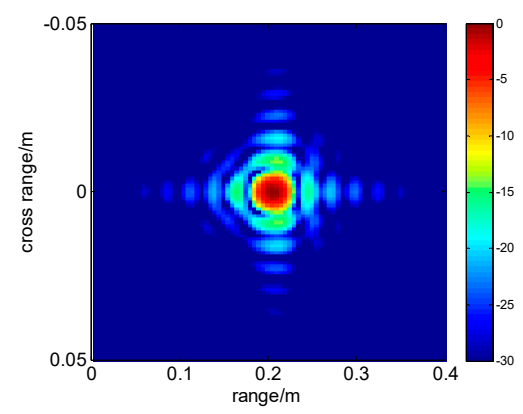

(b)

Figure 7. The array calibration factor and the imaging result after calibration, (a) is the array calibration factor and (b) is the imaging result after calibration.

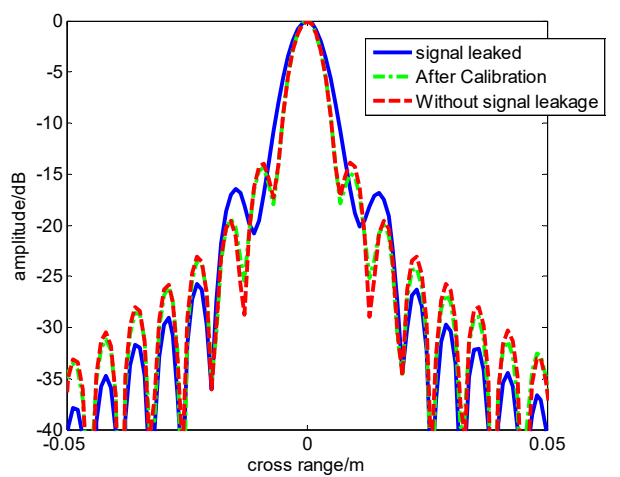

(a)

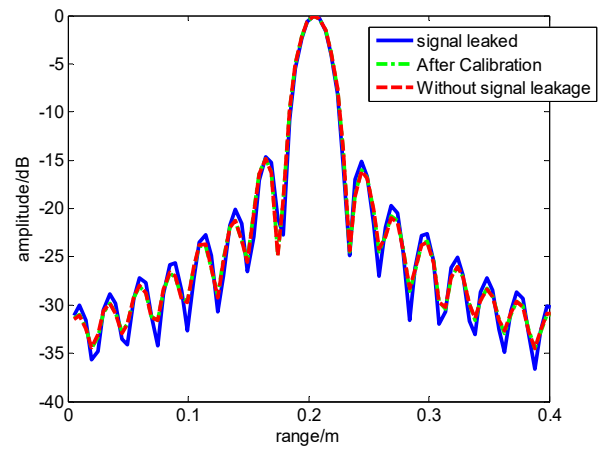

(b)

Figure 8. The PSF comparison of three different cases, (a) is the cross range PSF and (b) is the range PSF.

On the other hand, it can be found that the calibration factor is similar to a window function. Thus, the calibration process can be seen as an inverse process of windowing in the frequency domain. We can use the window function to replace the array calibration factor. We call this method the window function calibration method (WFCM). A Kaiser window with beta of 2.5 created by MATLAB shown in Figure $9 \mathrm{a}$ is used and the calibration result is shown in Figures $9 \mathrm{~b}$ and 10. 


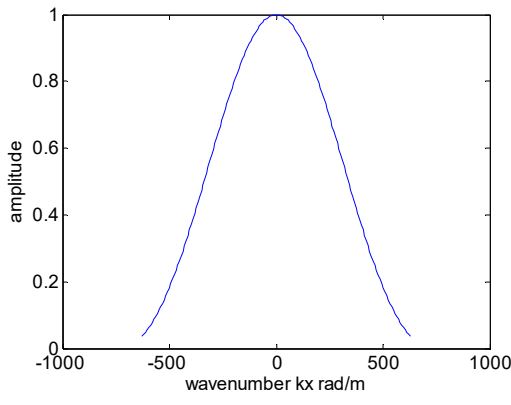

(a)

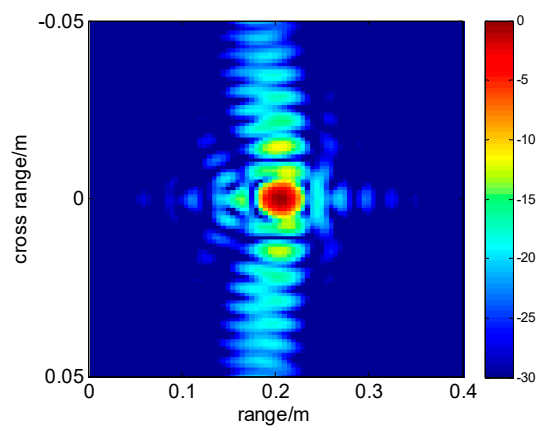

(b)

Figure 9. The Kaiser window with beta of 2.5 and its calibration result, (a) is Kaiser window and (b) is the calibration result.

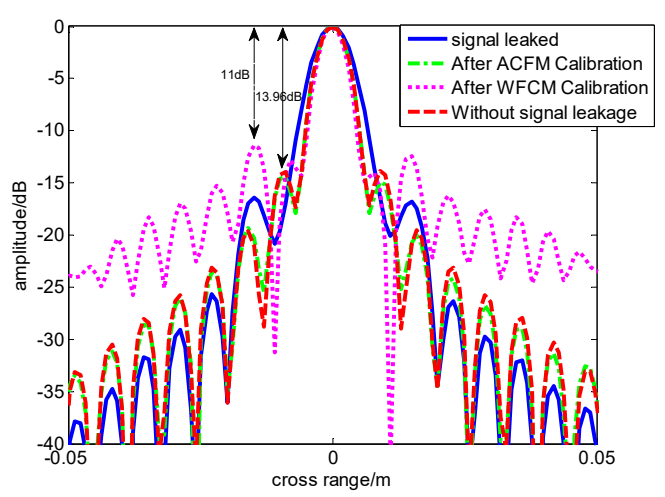

(a)

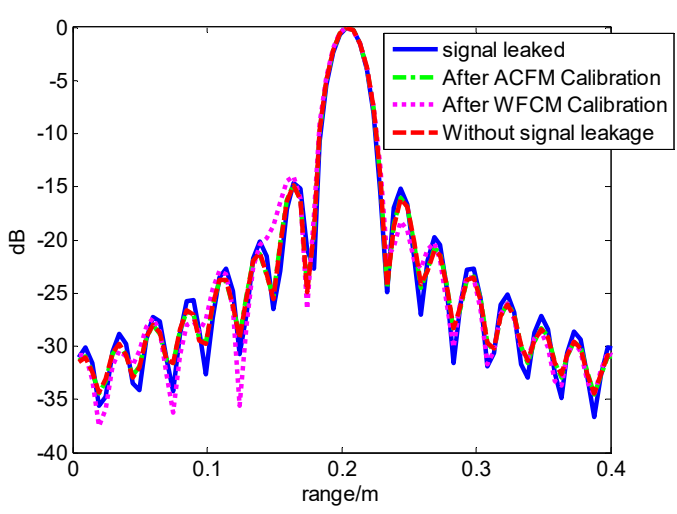

(b)

Figure 10. The PSF comparison of different cases in cross range and range, (a) is the cross range PSF and $(\mathbf{b})$ is the range PSF.

It can be seen that both methods can eliminate the broadening of the main lobe in the cross range. However, the side lobes raise evidently when using the WFCM method at the cross direction compared to the ACFM method described in Expression (11). It indicates that the array calibration factor created by Expression (11) can improve the image quality compared to the general window function. Impulse response width (IRW) and peak side lobe ratio (PSLR) [33] can be used to evaluate the calibration performance. The comparison of two calibration method performance is shown in Table 1. IRW is normalized with its theoretical resolution, so its unit is 1 . The measurement results of PSLR are also marked in Figure 10.

Table 1. The comparison of two calibration method performance.

\begin{tabular}{clc}
\hline Calibration Method & IRW & PSLR (dB) \\
\hline Array Calibration Factor Method (ACFM) & 1.008 & -13.96 \\
\hline Window Function Calibration Method (WFCM) & 1.004 & -11 \\
\hline
\end{tabular}

\subsection{Experiment Results}

An experiment was conducted to verify the effectiveness of the method mentioned above. The real array, targets, and photo of measurements in the experiments are shown in Figure 11. The configuration of the switch array is $\mathrm{K}=5, \mathrm{M}=4$, and $\mathrm{N}=4$. The operating frequency of the system is $27-33 \mathrm{GHz}$. The isolation of the array between different channels is about $25 \mathrm{~dB}$. A two-dimension (2D) imaging was carried out with a ball shown in Figure 11, which can be seen as a point target. The results are shown in Figure 12. Figure 12a is the 2D imaging result without calibration. Figure $12 \mathrm{~b}$ is the imaging result 
after calibration. Figure 13a,b are the PSF comparisons between the results before and after calibration. Obviously, it can be seen that the calibration process has an improvement on the cross-range resolution and has no impact on the range resolution. One point to note is that the calibration process improves the cross resolution and also raises the sidelobe levels. However, a set of calibration factors which is designed according to the structure of the array can bring as little side-lobe increases as possible compared to other window functions.

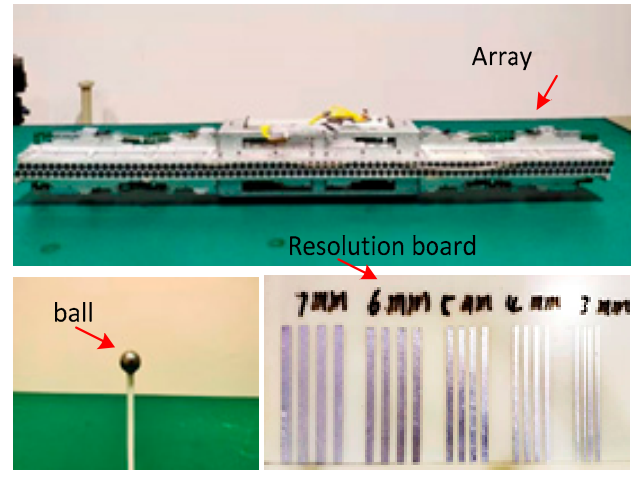

(a)

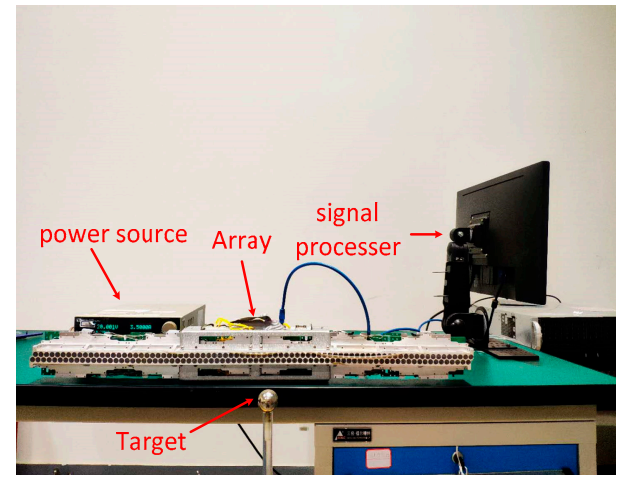

(b)

Figure 11. The array, targets and photo of measurements in experiments, (a) is array and targets; (b) is the experiment scene.

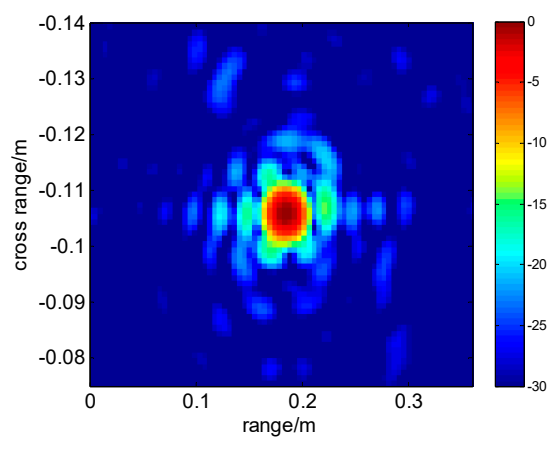

(a)

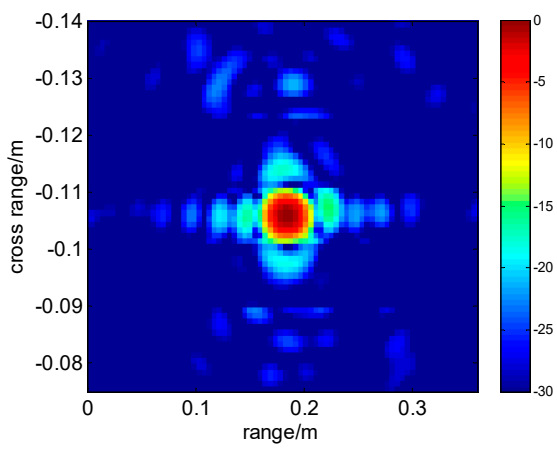

(b)

Figure 12. 2D imaging result of the ball in experiments, (a) is the imaging result before calibration and (b) is the imaging result after calibration.

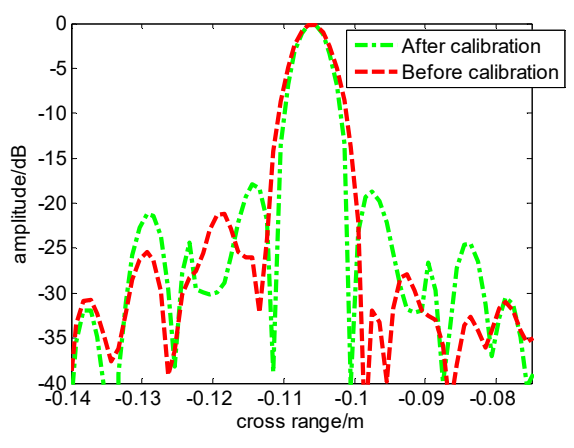

(a)

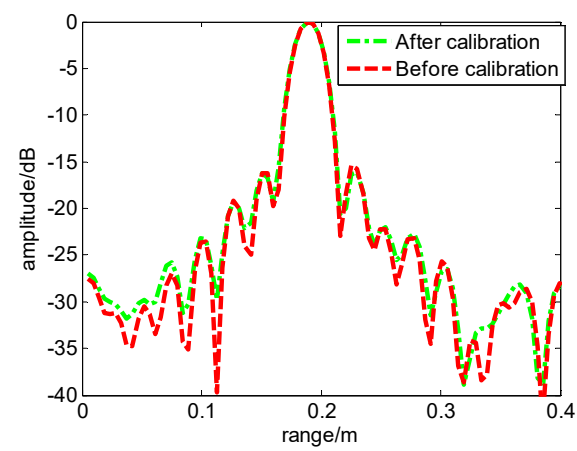

(b)

Figure 13. The PSF comparisons of the ball in experiments before and after calibration, (a) is the cross range PSF and (b) is the range PSF.

A simple resolution test board shown in Figure 11 was tested with three-dimension (3D) imaging. The maximum projection results are shown in Figure 14. Figure 14a shows the result without calibration. 
Figure $14 \mathrm{~b}$ shows the result calibrated with the method mentioned above. We can see that the gap between the aluminum bars in Figure 14b is clearer than the gap in Figure 14a. This shows that the calibration method can eliminate most of the impacts of the signal leakage and improve the cross resolution of the system.

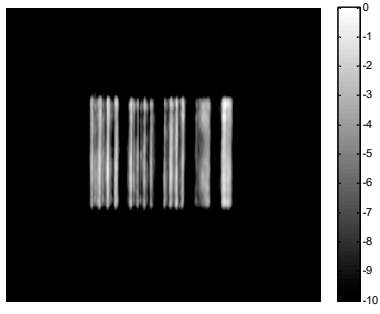

(a)

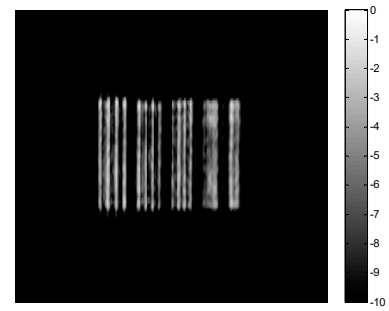

(b)

Figure 14. Imaging results of the resolution board in experiments before and after calibration, (a) is the imaging result before calibration and (b) is the imaging result after calibration.

In this section, a simulation and some experiments are carried out to validate the effectiveness of the proposed method. The simulation and the experiment results indicate that this calibration method can mostly cancel the influence of the signal leakages.

\section{Conclusions}

This paper discussed the influence of the signal leakages between different channels in the application of array imaging. Signal leakage between different channels can deteriorate the system cross resolution but has no impact on the range resolution. When the channel isolation is not good enough, it will lead to the appearance of grating lobes, which we tried to eliminate in this paper. A calibration method was presented to eliminate the influences of signal leakages and was validated with the simulation and some experiments. The calibration factor proposed in this paper can better improve the image quality compared to the used window function. However, we have not yet obtained an optimal window function selection method which may obtain better calibration results. Some optimization methods can be tried in related directions. It will be carried out in future work. The effect of noise on the calibration process proposed in the paper also can be a research direction in the next work. The same analysis method and calibration method can be extended to complex array imaging application analyses.

Author Contributions: Conceptualization, Z.C.; methodology, Z.C.; software, Q.W.; validation, J.A., and Y.Y.; formal analysis, Z.C.; writing-original draft preparation, Z.C.; writing-review and editing, B.C.; project administration, B.C.; funding acquisition, X.D. All authors have read and agreed to the published version of the manuscript.

Funding: This research was funded by National Key R\&D Program of China Grant (2018YFB1801504), the National Natural Science Foundation of China Grant (61805217) and the President Funding of China Academy of Engineering Physics with No. YZJJLX2018009.

Conflicts of Interest: The authors declare no conflict of interest.

\section{References}

1. Sheen, D.M.; Clark, R.T.; Tedeschi, J.R.; McCall, J.; Hartman, T.S.; Jones, A.M. Efficient image reconstruction method for a millimeter-wave shoe scanner. In Proceedings of the Passive and Active Millimeter-Wave Imaging XXIII, Vancouver, BC, Canada, 23 April 2020.

2. Jones, M.; Sheen, D.; Tedeschi, J. Wideband archimedean spiral antenna for millimeter-wave imaging array. In Proceedings of the 2017 IEEE International Symposium on Antennas and Propagation \& USNC/URSI National Radio Science Meeting, San Diego, CA, USA, 9-15 July 2017; pp. 845-846. 
3. Sheen, D.; Jones, M.; Hall, T.E. Simulation of active cylindrical and planar millimeter-wave imaging systems. In Proceedings of the Passive and Active Millimeter-Wave Imaging XXI, Orlando, FL, USA, 18-20 April 2018.

4. Meng, Y.; Lin, C.; Zang, J.; Qing, A. MMW Holographic Imaging for Security Check. In Proceedings of the 2019 IEEE Asia-Pacific Microwave Conference (APMC), Singapore, 10-13 December 2019; pp. 569-571.

5. Ma, Q.; Chung, H.; Rebeiz, G.M. A 4-Channel 10-40 GHz Wideband Receiver with Integrated Frequency Quadrupler for High Resolution Millimeter-Wave Imaging Systems. In Proceedings of the 2018 IEEE/MTT-S International Microwave Symposium-IMS, Philadelphia, PA, USA, 10-15 June 2018; pp. 883-886.

6. Cheng, B.; Cui, Z.; Lu, B.; Qin, Y.; Liu, Q.; Chen, P.; He, Y.; Jiang, J.; He, X.; Deng, X.; et al. 340-GHz 3-D Imaging Radar With 4Tx-16Rx MIMO Array. IEEE Trans. Terahertz Sci. Technol. 2018, 8, 509-519. [CrossRef]

7. Li, S.; Zhao, G.-Q.; Sun, H.; Amin, M.G. Compressive Sensing Imaging of 3-D Object by a Holographic Algorithm. IEEE Trans. Antennas Propag. 2018, 66, 7295-7304. [CrossRef]

8. Gao, J.; Qin, Y.; Beng, B.; Wang, H.; Li, X. A novel method for 3D milliter-wave holographic reconstruction based on frequency interferometry techniques. IEEE Trans. Microw. Theory Technol. 2018, 66, 1579-1596. [CrossRef]

9. Gao, J.; Qin, Y.; Deng, B.; Wang, H.; Li, X. Novel Efficient 3D Short-Range Imaging Algorithms for a Scanning 1D-MIMO Array. IEEE Trans. Image Process. 2018, 27, 3631-3643. [CrossRef] [PubMed]

10. Gao, J.; Deng, B.; Qin, Y.; Li, X.; Wang, H. Point Cloud and 3-D Surface Reconstruction Using Cylindrical Millimeter-Wave Holography. IEEE Trans. Instrum. Meas. 2019, 68, 4765-4778. [CrossRef]

11. Mirbeik-Sabzevari, A.; Oppelaar, E.; Ashinoff, R.; Tavassolian, N. High-Contrast, Low-Cost, 3-D Visualization of Skin Cancer Using Ultra-High-Resolution Millimeter-Wave Imaging. IEEE Trans. Med. Imaging 2019, 38, 2188-2197. [CrossRef] [PubMed]

12. Mirbeik-Sabzevari, A.; Li, S.; Garay, E.; Nguyen, H.T.; Wang, H.; Tavassolian, N. Synthetic Ultra-High-Resolution Millimeter-Wave Imaging for Skin Cancer Detection. IEEE Trans. Biomed. Eng. 2019, 66, 61-71. [CrossRef] [PubMed]

13. Mirbeik-Sabzevari, A.; Tavassoian, N.; Ashinoff, R. Ultra-High-Resolution Millimeter-Wave Imaging: A New Promising Skin Cancer Imaging Modality. In Proceedings of the 2018 IEEE Biomedical Circuits and Systems Conference (BioCAS), Cleveland, OH, USA, 17-19 October 2018; pp. 1-4.

14. Wang, P.; Millar, D.; Parsons, K.; Orlik, P.V. Nonlinearity correction for range estimation in FMCW millimeter-wave automotive radar. In Proceedings of the 2018 IEEE MTT-S International Wireless Symposium (IWS), Chengdu, China, 6-10 May 2018; pp. 1-3.

15. Wang, P.; Millar, D.; Parsons, K.; Ma, R.; Orlik, P.V. Range Accuracy Analysis for FMCW Systems with Source Nonlinearity. In Proceedings of the 2019 IEEE MTT-S International Conference on Microwaves for Intelligent Mobility (ICMIM), Detroit, MI, USA, 15-16 April 2019; pp. 1-5.

16. Li, Y.; Hu, W.; Zhang, X.; Zhao, Y.; Ni, J.; Ligthart, L.P. A Non-Linear Correction Method for Terahertz LFMCW Radar. IEEE Access 2020, 8, 102784-102794. [CrossRef]

17. Schiessl, A.; Genghammer, A.; Ahmed, S.S.; Schmidt, L.P. Phase error sensitivity in multistatic microwave imaging systems. In Proceedings of the 43rd European Microwave Coference, Nuremberg, Germany, 7-10 October 2013; pp. 1631-1634.

18. Pinel-Puyssegur, B.; Lasserre, C.; Benoit, A.; Jolivet, R.; Doin, M.; Champenois, J. A Simple Phase Unwrapping Errors Correction Algorithm Based on Phase Closure Analysis. In Proceedings of the IGARSS 2018-2018 IEEE International Geoscience and Remote Sensing Symposium, Valencia, Spain, 23-27 July 2018; pp. 2212-2215.

19. Deng, H.; Farquharson, G.; Sahr, J.; Goncharenko, Y.; Mower, J. Phase Calibration of an Along-Track Interferometric FMCW SAR. IEEE Trans. Geosci. Remote Sens. 2018, 56, 4876-4886. [CrossRef]

20. Korner, G.; Oppelt, D.; Adametz, J.; Vossiek, M. Novel Passive Calibration Method for Fully Polarimetric Near Field MIMO Imaging Radars. In Proceedings of the 2019 12th German Microwave Conference (GeMiC), Stuttgart, Germany, 25-27 March 2019.

21. Zhao, Q.; Zhang, Y.; Wang, W.; Wang, P.; Wang, R.; Deng, Y.; Zhang, H.; Ye, K.; Zhou, Y. Channel Imbalance Compensation with IF Signal for China's IDBSAR. In Proceedings of the IGARSS 2019-2019 IEEE International Geoscience and Remote Sensing Symposium, Yokohama, Japan, 28 July-2 August 2019; pp. 1112-1115.

22. Shin, D.H.; Jung, D.H.; Kim, D.C.; Ham, J.W.; Park, S.O. A distributed FMCW radar system based on Fiber-Optic links for small drone decetion. IEEE Trans. Instrum. Meas. 2017, 66, 340-347. [CrossRef] 
23. Lee, S.J.; Jung, J.H.; Yu, J.-W. TX Leakage Canceller for Small Drone Detection Radar in 2-GHz Band. IEEE Microw. Wirel. Compon. Lett. 2020, 30, 213-215. [CrossRef]

24. Melzer, A.; Onic, A.; Huemer, M. Self-Adaptive Short-Range Leakage Canceler for Automotive FMCW Radar Transceivers. In Proceedings of the 2018 15th European Radar Conference (EuRAD), Madrid, Spain, 26-28 September 2018; pp. 26-29.

25. Bauduin, M.; Bourdoux, A. Mixed-Signal Transmitter Leakage Cancellation for PMCW MIMO Radar. In Proceedings of the 2018 15th European Radar Conference (EuRAD), Madrid, Spain, 26-28 September 2018; pp. 293-297.

26. Arnold, B.T.; Jensen, M.A. The Effect of Antenna Mutual Coupling on MIMO Radar System Performance. IEEE Trans. Antennas Propag. 2019, 67, 1410-1416. [CrossRef]

27. Colon-Diaz, N.; McCormick, P.M.; Janning, D.; Bliss, D.W. Compensation of Mutual Coupling Effects for Co-located MIMO Radar Applications via Waveform Design. In Proceedings of the 2019 International Radar Conference (RADAR), Toulon, France, 23-27 September 2019; pp. 1-6.

28. Chen, X.; Zhang, S.; Li, Q. A Review of Mutual Coupling in MIMO Systems. IEEE Access 2018, 6, $24706-24719$. [CrossRef]

29. Sheen, D.; McMakin, D.; Hall, T. Three-dimensional millimeter-wave imaging for concealed weapon detection. IEEE Trans. Microw. Theory Tech. 2001, 49, 1581-1592. [CrossRef]

30. Kell, R. On the derivation of bistatic RCS from monostatic measurements. Proc. IEEE 1965, 53, 983-988. [CrossRef]

31. Stolt, R.H. Migration by Fourier Transform. Geophysics 1978, 43, 23-48. [CrossRef]

32. Jiacheng, S.; Chen, M. Research on Imaging Algorithm of Millimeter Wave Radar Based on Stolt Interpolation. In Proceedings of the 2019 IEEE MTT-S International Microwave Biomedical Conference (IMBioC), Nanjing, China, 6-8 May 2019; pp. 1-4.

33. Cumming, I.G.; Wong, F.H. Digital Processing of Synthetic Aperture Radar Data Algorithms and Implementation; Artech House: Boston, MA, USA, 2005; pp. 39-41.

(C) 2020 by the authors. Licensee MDPI, Basel, Switzerland. This article is an open access article distributed under the terms and conditions of the Creative Commons Attribution (CC BY) license (http://creativecommons.org/licenses/by/4.0/). 